\title{
A new approach involving a multi transducer ultrasonic system for cleaning turbine engines' oil filters under practical conditions
}

\author{
Dinh Duc Nguyen ${ }^{\mathrm{a}, \mathrm{b}}$, Huu Hao Ngo ${ }^{\mathrm{c}}$, Yong Soo Yoon ${ }^{\mathrm{d}}$, Soon Woong Chang ${ }^{\mathrm{a}}$, Hong Ha Bui ${ }^{\mathrm{e}}$ \\ ${ }^{a}$ Department of Environmental Energy \& Engineering, Kyonggi University, 443-760, Republic of \\ Korea \\ ${ }^{\mathrm{b}}$ Ho Chi Minh City University of Natural Resources and Environment, Viet Nam \\ ${ }^{\mathrm{c}}$ Centre for Technology in Water and Wastewater, School of Civil and Environmental Engineering, \\ University of Technology, Sydney (UTS), 15 Broadway, Ultimo, NSW 2007, Australia \\ ${ }^{d}$ Department of Chemical Engineering, Dankook University, 448-701, Republic of Korea \\ ${ }^{\mathrm{e}}$ Institute for Tropicalization and Environment (ITE), Ho Chi Minh City, Viet Nam
}

\section{Highlights}

- A new approach for large-scale oil filter cleaning of turbine engines was examined in detail.

- Significant reduction of oil filter fouling was achieved through ultrasonic cleaning.

- Ultrasonic cleaning has been demonstrated as cleaner, faster, easier and safer than manual washing.

- Ultrasonic cleaning efficiency was significantly affected by solvent using.

- Ultrasound disruption of foulant particles deposited in the oil filter was successful.

\begin{abstract}
The purpose of this paper is to provide a green technology that can clean turbine engine oil filters effectively in ships using ultrasound, with ultrasonic devices having a frequency of $25 \mathrm{kHz}$ and different powers of $300 \mathrm{~W}$ and $600 \mathrm{~W}$, respectively. The effects of temperature, ultrasonic cleaning times, pressure losses through the oil filter, solvent washing, and ultrasonic power devices were investigated. In addition, the cleaning efficiency of three modes (hand washing, preliminary washing and ultrasonic washing) were compared to assess their relative effectiveness. Experimental results revealed that the necessary ultrasonic time varied significantly depending on which solvent was used for washing. For instance, the optimum ultrasonic cleaning time was 50-60 min when the oil filter was cleaned in a solvent of kerosene oil (KO) and over $80 \mathrm{~min}$ when in a solvent of diesel oil (DO) using the same ultrasonic generator device $(25 \mathrm{kHz}, 600 \mathrm{~W})$ and experimental conditions. Furthermore, microscopic examination did not reveal any damage or breakdown on or within the structure of the filter after ultrasonic cleaning, even in the filter's surfaces at a constantly low frequency of $25 \mathrm{kHz}$ and power specific capacity (100 W/gal). Overall, it may be concluded that ultrasound-assisted oil filter washing is effective, requiring a significantly shorter time than manual washing. This ultrasonic method also shows promise as a green technology for washing oil filters in turbine engines in general and Vietnamese navy ships in particular, because of its high cleaning efficiency, operational simplicity and savings.
\end{abstract}




\section{Keywords}

Ultrasonic devices; Turbine engines; Ultrasonic cleaning; Oil filter; Ultrasonic irradiation

\section{Introduction}

A turbine engine is a type of internal combustion engine used widely in factories, power plants, planes, ships, tanks, trains, etc. Engines are composed of very complex, detailed mechanical elements requiring high performance accuracy. Therefore, lubricating oil, hydraulic oil and fuel oil in turbine engines must always be of high quality and filtered through a small metal filter system to ensure all impurities arising during operations are eliminated [1], [2] and [3].

In recent years, the Vietnamese naval forces have been progressively equipped with modern facilities to improve sea defences and combat training; the majority of these modern ships use turbine engines. One of the factors affecting the sustainability and productivity of turbine engines is the quality of fuel oil, lubricant oil, hydraulic oil, etc. When a turbine engine is running, fuel oil, lubricating oil and hydraulic oil are sent continuously through the oil filter system to ensure all impurities arising in the course of work are removed, so the engine can continue functioning normally.

Over time, the oil filter is clogged by impurities (oversized particulates or products) that have been filtered out of the oil and by a wide variety of foulants, even the activities of microorganisms [3] and [4]. In general, these impurities significantly reduce the productivity of the filter device. Thus, in order to stabilise the productivity of the device, it is necessary to replace or clean the soiled oil filter at regular intervals. To clean the oil filter, either of these two common methods is generally applied: (1) cleaning with solvent combined with manual washing or (2) cleaning with solvent combined with ultrasound waves.

The process of conventional manual cleaning with solvent often uses strongly polarized solvents such as those containing halogen atoms, for example methyl halide compounds[3] and [5]. Utilising these compounds is not only corrosive to filters and detrimental to the surrounding environment, and for the mechanic, but conventional manual cleaning also wastes a large quantity of solvent. Thus from this perspective, ultrasonic cleaning - which uses smaller quantities of less-corrosive solvent - would be a more suitable method than conventional manual cleaning.

In recent years, research applications of ultrasonic waves to clean surface materials (in industry, medicine, and laboratory fields) are attracting the attention of many scientists around the world as an innovative technology and a priority for further research and development [6], [7], [8] and [9]. The ultrasonic process generates numerous advantageous physical and chemical phenomena, like shear forces, micro-jets, micro-streamings, shock waves, and free radical species (OH, H, O, etc.) [10], [11], [12],[13] and [14], and in particular creates a series of tiny and transient cavitation bubbles with a diameter of less than $100 \mu \mathrm{m}$ [11]; these tiny bubbles penetrate nearly every corner and crevice of the filter which conventional cleaning methods cannot reach or easily access [9]. These cavitation bubbles exist for an extremely short (transient supercritical) time, then are violently collapsed during the compressional phase, so that they emit shock waves [10] and [15] with high temperatures (up to $5000 \mathrm{~K}$ ) and pressures (up to $1000 \mathrm{~atm}$ ) [7], [12] and [16]. This microscopic process acts with sufficient energy on the dirt surfaces (soluble contaminants and insoluble particles), that they gradually separate and dissolve into the solvent solution from the filter, leading to complete filter cleaning. Conversely, manual cleaning such as by air spray and brush cannot achieve such complete cleaning [9]. Essentially, the main benefits of the ultrasonic cleaning 
method include better efficiency, shortened filter cleaning time, safety, easy installation, simple operation and maintenance, and reduced labour costs, despite the slightly higher electrical power consumption.

Several studies have been conducted on the application of ultrasonic waves to clean the surface of materials, such as cleaning the surface of solar cell panels [17] and [18], cleaning the membranes in water treatment [6], [12] and [19], and cleaning motor and sailing boats at the ferry terminal [20]. All this research has achieved excellent results at a very reasonable cost. To minimise harm to the environment, to protect personnel from the health risks of manual washing, and especially to achieve highly efficient cleaning, ultrasonic cleaning devices have generated much interest and are worth investigating for oil filter cleaning.

To date the most common filter cleaning work on navy ships in Vietnam is still done manually. Overall, the traditional and current filter cleaning procedure is quite complicated, costly and time-consuming, resulting in poor efficiency and uneven results. (Technicians remove the filter, place it in clean kerosene oil (KO) or diesel oil (DO) as a solvent, and brush with a soft brush over the filter surface; then the filter is rinsed off with new KO or DO, followed by the technicians using compressed air to spray the filter after every scouring in new KO or DO. This work is repeated until the KO or DO that is rinsed out is no longer dirtstained, then the filter is considered clean and reassembled.) This method may cause oil filter damage and consume large quantities of solvent. In addition, manual scouring with uneven force may damage the filter, clean only the surfaces, or cause secondary contaminations.

In recent years only very few Vietnam navy ships were equipped with ultrasonic filter cleaning equipment, but there are still many shortcomings in the operation and optimal efficiency of the devices according to local conditions. It is suggested that the cleaning of fouled oil filters by ultrasound in association with solvent oils (KO or DO) is a novel method that can clean turbine engine oil filters at a reasonable cost.

Currently, Vietnam is carrying out renovations and building new navy ships, using turbine engines, which are equipped with ultrasonic oil filter cleaning system. Studying the ultrasonic cleaning process as micro-level cleaning, designing the right equipment, and improving cleanliness in washing procedures as well as inspection procedures are imperative to significantly reduce the foulants doing damage to the turbine engine's efficiency.

Therefore, the present study was conducted to determine the following: (1) the procedure and method of cleaning the oil filter using ultrasound equipment, (2) the better solvent for ultrasonic cleaning, (3) the ideal duration, intensity, and frequency $(\mathrm{kHz})$ of ultrasound exposure, (4) the parameters for evaluating oil filter cleanliness in turbine engines, and (5) any possible changes in the solvent oils after ultrasonic irradiation.

\section{Materials and methods}

\subsection{Experimental design and set-up}

\subsubsection{Subject of the study}

The experiments in this study were conducted on two types of oil filters (lubricant oil filter and fuel oil filter) used in navy ships' turbine engines (Fig. 1), and on oil filters used in antisubmarine ships of Brigade 171, Navy Region X, Vietnam. The fine-scale filters were micro filters with pore sizes ranging from 10 to $16 \mu \mathrm{m}$, made of copper or stainless steel in circular disc-shapes, with $25 \mathrm{~mm}$ inside diameter, $70 \mathrm{~mm}$ outside diameter, and thickness $5 \mathrm{~mm}$ (Fig. $1)$. 

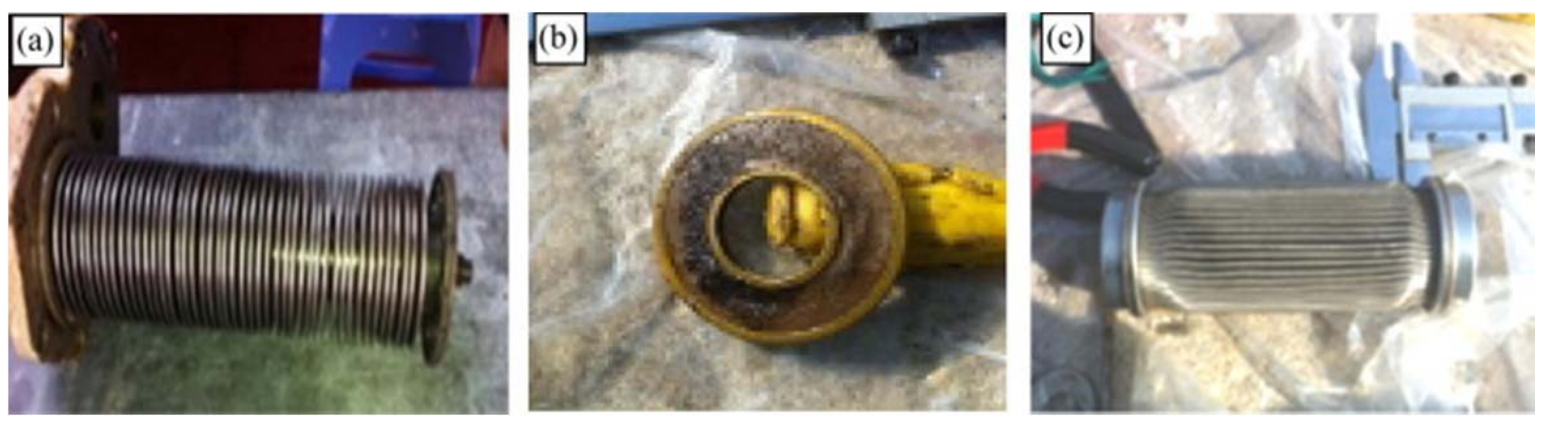

Fig. 1. Fouling of fuel oil filters (a) and lubricating oil filters (b, c) in turbine engines.

\subsubsection{Experimental apparatus}

Ultrasonic wave experiments were conducted with two ultrasonic systems (a bath-type sonoreactor model). Both ultrasound devices had an oscillated frequency of $25 \mathrm{kHz}$; the Model MU-300 had a power of $300 \mathrm{~W}$, and the Model MU-600 had a power of $600 \mathrm{~W}$ (Mirae Ultrasonic Tech. Co. Ltd., South Korea) (Fig. 2a). The models MU-300 and MU-600 were equipped with 6 transducers and 12 transducers, respectively, and were arranged and fixed under the bottom and two sides of the reactor. The transducer diameter was $60 \mathrm{~mm}$ (Mirae Ultrasonic Tech. Co. Ltd., South Korea).
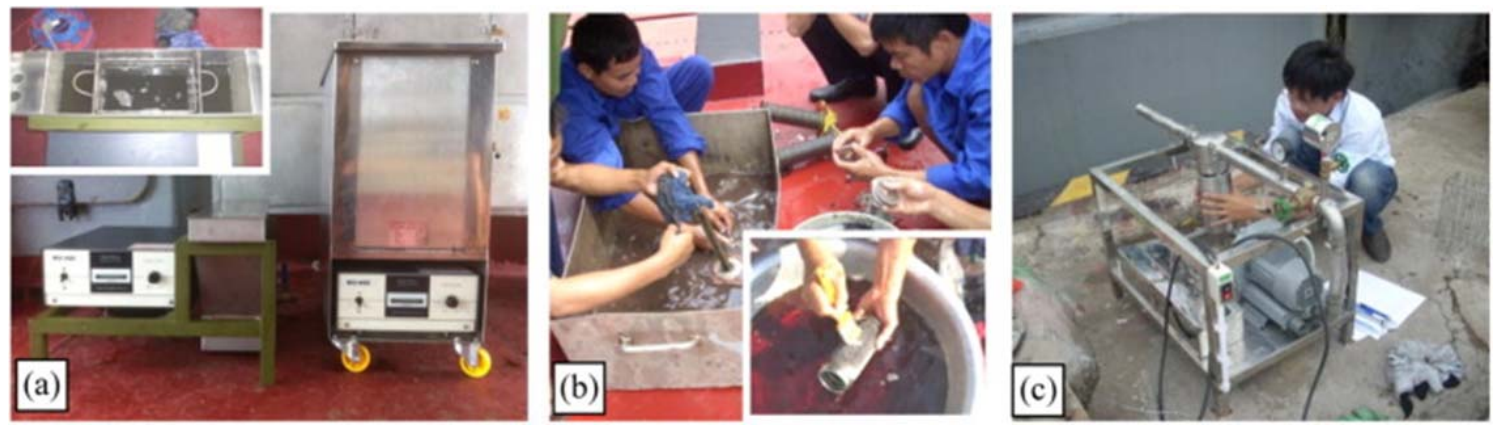

Fig. 2. Ultrasonic cleaning system (a), manual cleaning (b) and device for measuring the pressure drop (c).

The air velocity and degree of pressure loss through the filters (before and after each test) of the experiments were measured by VITTEP 01, which had a power of 0.5HP (VITTEP, Vietnam) (Fig. 2c), and by Testo 350 XL (Ashtead Technology Co., Ltd.) in order to determine the effectiveness of the cleaning procedure.

\subsubsection{Solvents and sampling}

The solvents used in these experiments to fill the cleaner tank were industrial kerosene oil $(\mathrm{KO})$, and diesel oil (DO), which are widely used in various industries today. The main properties of $\mathrm{KO}$ and DO are summarised in Table 1 and Table 2. 
Table 1. Characteristics of kerosene oil.

\begin{tabular}{llcc}
\hline No. & Parameter & KO & Test method \\
\hline $\mathbf{1}$ & Flash point PMCC,${ }^{\circ} \mathrm{C}$ & 38 & {$[27,28]$} \\
$\mathbf{2}$ & Distillation of KO at $0^{\circ} \mathrm{C}$ & & {$[29]$} \\
& $-10 \%$ of volume, max & 300 & \\
& - End boiling point, max & 0.30 & {$[30,31]$} \\
$\mathbf{3}$ & A maximum sulfur content, percent by mass & 19 & {$[32]$} \\
$\mathbf{4}$ & Smoke point, mm & Type III & {$[33]$} \\
$\mathbf{5}$ & Copper strip corrosion, $3 \mathrm{~h}$ at $100^{\circ} \mathrm{C}$ & $1.0-1.9$ & {$[34]$} \\
$\mathbf{6}$ & The kinematic viscosity in cSt at $40^{\circ} \mathrm{C}$ & Negative & {$[35]$} \\
$\mathbf{7}$ & Detection of mercaptans, hydrogen sulfide & - & {$[36]$} \\
$\mathbf{8}$ & Density at $15^{\circ} \mathrm{C}, \mathrm{kg} / 1$ & & \\
\hline
\end{tabular}

PMCC: Pensky-Martens closed cup.

Table 2. Characteristics of diesel fuel oil.

No. Product property

1 Sulfur content, $\mathrm{mg} / \mathrm{kg}$, max

2 Distillation, ${ }^{\circ} \mathrm{C}, 90 \%$ vol recovered, $\max$

$3 \quad$ Flash point, ${ }^{\circ} \mathrm{C}$, min

$4 \quad$ Vicosity at $40^{\circ} \mathrm{C}, \mathrm{cSt}$, min-max

5 Pour point, ${ }^{\circ} \mathrm{C}, \max$

6 Corrosion, Copper strip, classification, max (3h at $\left.50^{\circ} \mathrm{C}\right)$

$7 \quad$ Density at $15^{\circ} \mathrm{C}, \mathrm{kg} / \mathrm{m} 3, \min -\max$

\begin{tabular}{ccc}
$\begin{array}{c}\text { Diesel fuel oil } \\
\text { type }\end{array}$ & $\begin{array}{c}\text { Test } \\
\text { method }\end{array}$ \\
\cline { 1 - 2 } $\begin{array}{c}\text { DO } \\
0.05 \%\end{array}$ & $\begin{array}{c}\text { DO } \\
\text { S }\end{array}$ & \\
S & & \\
500 & 2500 & {$[30,31,37]$} \\
360 & 360 & {$[29]$} \\
55 & 55 & {$[27,38]$} \\
$2-4.5$ & $2-4.5$ & {$[34,39]$} \\
+6 & +6 & {$[40,41]$} \\
Type I & Type I & {$[33]$} \\
& & \\
$820-$ & $820-$ & {$[36]$} \\
860 & 860 &
\end{tabular}




\subsection{Cleaning experiments}

\subsubsection{Experimental procedure}

The schematic block diagram (Fig. 3) represents the experimental procedure used to compare and optimise the oil filter cleaning process by means of ultrasound.

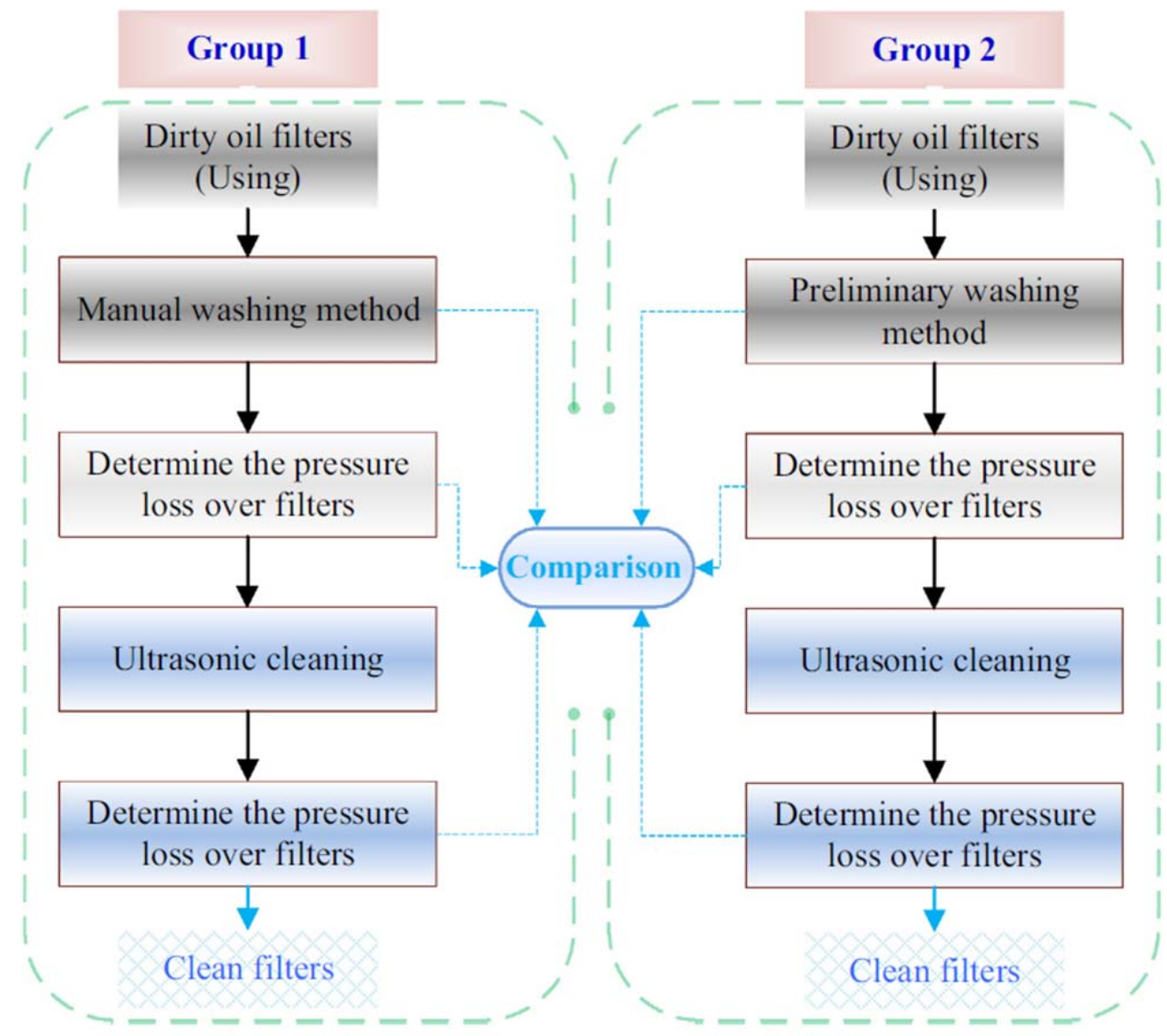

Fig. 3. A schematic block diagram of the oil filter cleaning process by ultrasonic waves.

\subsubsection{Experiment description}

The used and clogged oil filters of marine turbine engines (ship HQ-9X and ship HQ-17X) were removed from the filter housing; each filter housing contains 40 disc filters. The experiment was conducted on two groups, each with 20 disc filters. The filters were put into equipment measuring pressure losses, to determine the original overall pressure losses (Fig. 4).

The first group was cleaned by the method according to the first branch (Group 1) of the diagram in Fig. 3. The manual washing ${ }^{1}$ step was undertaken by a ship's mechanic (Fig. 2b). After standard manual washing, the filters were measured to obtain pressure loss values. Subsequently, the filters were ultrasonically cleaned for $60 \mathrm{~min}$ and then checked for pressure loss again. The second group of experiments was conducted according to the second branch of the diagram (Fig. 3). The filters were subjected to a preliminary washing ${ }^{2}$ that only removed the top layers of plaque deposited on the surface of the disc filter, and the overall pressure loss was measured. Then the filters were ultrasonically cleaned for $60 \mathrm{~min}$ and 
measured a second time for pressure loss. The results of the measured pressure losses after each type of cleaning were analysed, to evaluate the relative cleaning ability of these methods, manual washing and ultrasonic waves.

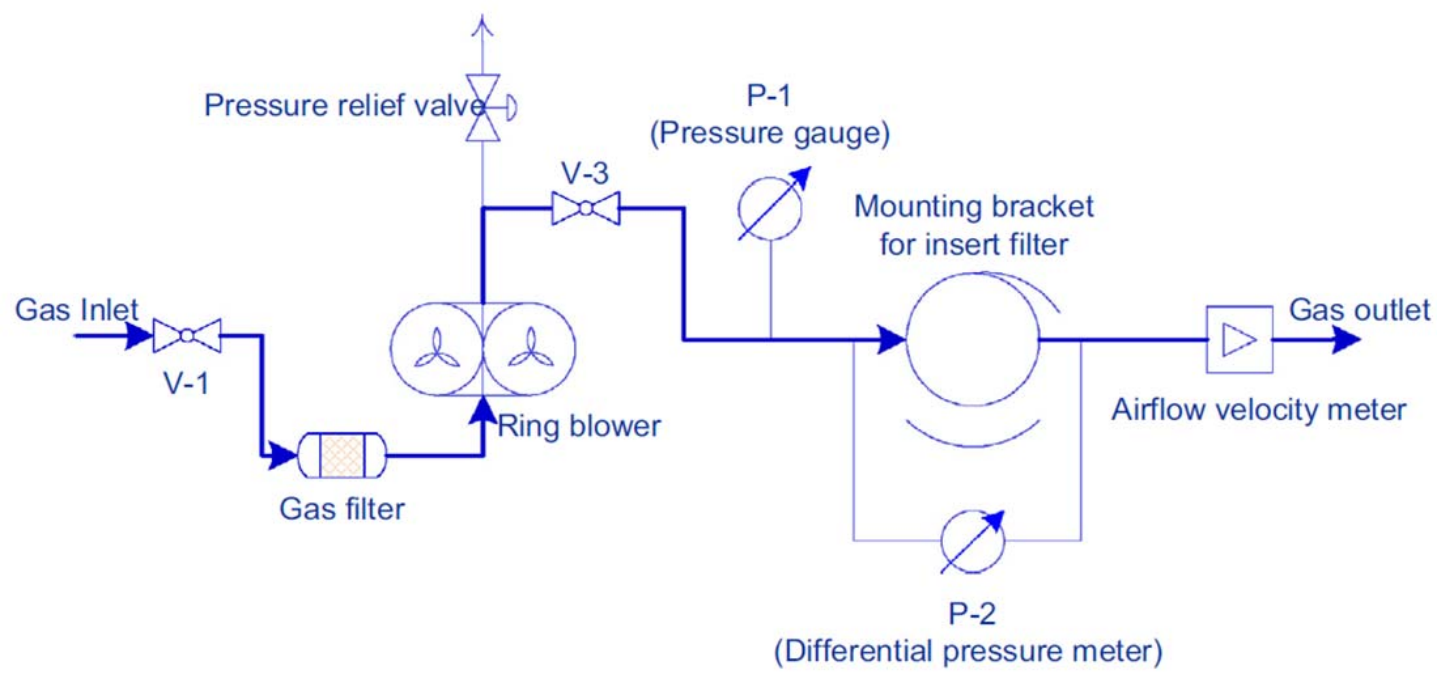

Fig. 4. Schematic diagram of filter cleanliness inspection.

\subsection{Sampling and analytical methods}

Oil samples (KO, DO) were taken before and after each ultrasonic cleaning, and the corrosion criteria of the thin copper foil were examined in order to evaluate the possibility that the solvent oils might be denatured by the ultrasound process, according to method ASTM D130[21].

During experiments, the parameters to be checked included the following: (1) the solvent temperature was measured directly by thermometer for each experiment, recorded once every 10 min over a duration of $90 \mathrm{~min}$; (2) the level of corrosion of the copper foil in the solvent was evaluated before and after ultrasonic irradiation; (3) Air velocity was measured directly by electronic gauges to determine gas flow through the filter; and (4) the pressure loss and velocity across the filter were measured during the experiment according to the diagram in Fig. 4.

Statistical analysis methods were also applied to evaluate the correlation between laboratory data and operating parameters, and this correlation was assessed by the level of significance $(p)$ and Pearson's correlation coefficient $(r)$.

\section{Results and discussion}

\subsection{Effect of solvents in washing}

The solvents used in ultrasonic cleaning play a very important role in cleaning technology [5] and [22], acting simultaneously as a medium for sound waves propagating and as a solution to dissolve contaminants. Hence, the relative properties and benefits of these various solvents should be closely studied, thereby increasing ultrasonic cleaning's reliability and effectiveness, leading to its more widespread application in various industries.

\subsubsection{Variation of temperature over ultrasonic irradiation time}


The temperatures of the solvents in the ultrasonic system were measured directly with thermometers and results automatically saved to the computer every $10 \mathrm{~min}$ during the 90 min exposure period.

The experimental results (Fig. 5) show that the values of the solvent temperature increase when the ultrasound irradiation time also increases. The explanation for this is that the ultrasound device transforms electrical power into thermal energy (heat) [23], which can result in cavities' violent collapse [18] and [24].

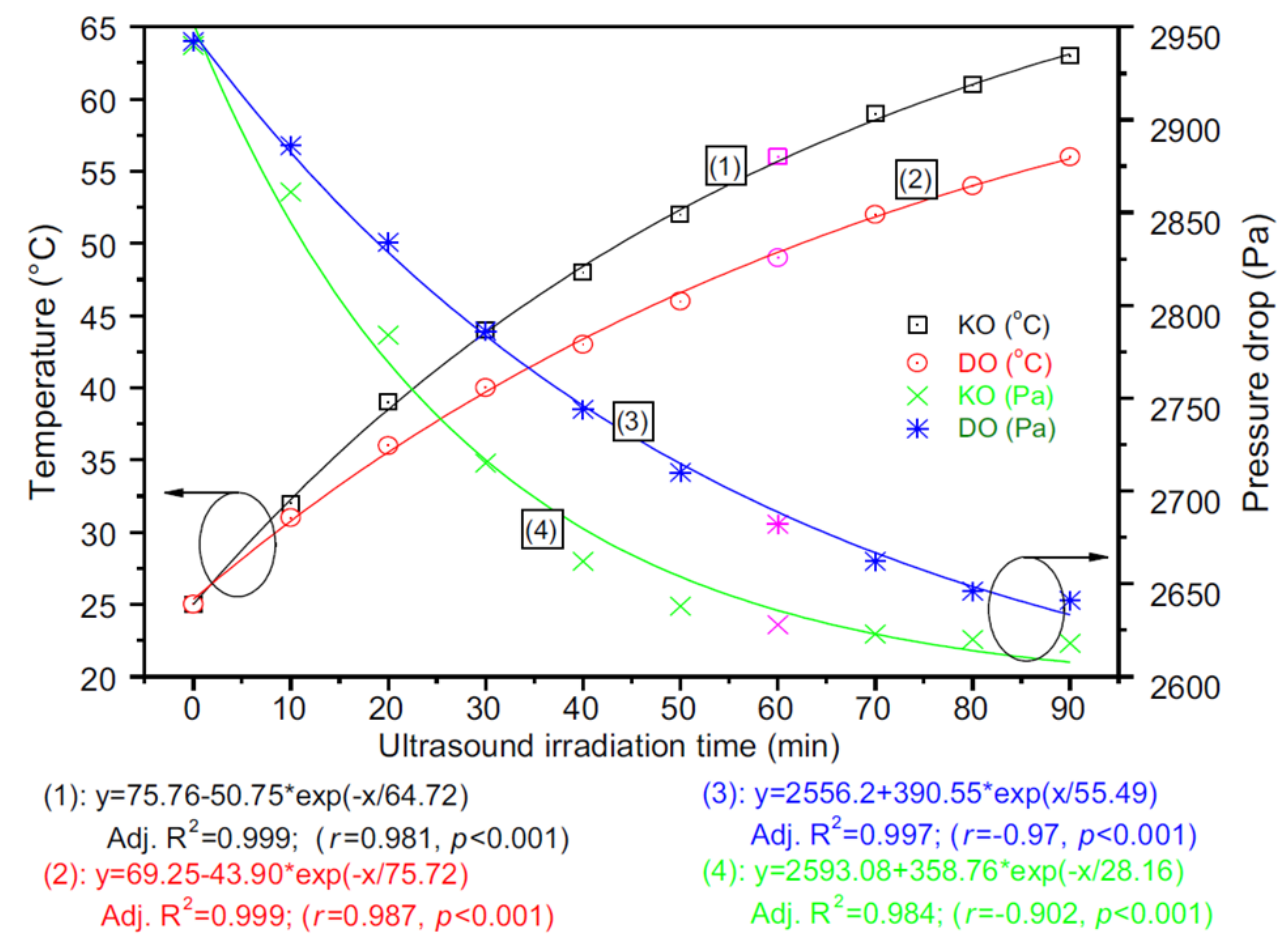

Fig. 5. The variation of temperature and pressure drop over ultrasound irradiation time.

At the initial operation time, the temperatures of both ultrasonic solvents were the same, approximately $25^{\circ} \mathrm{C}$. In the solvents of $\mathrm{DO}$ and $\mathrm{KO}$, after $90 \mathrm{~min}$ of ultrasound, the corresponding temperatures rose from $25^{\circ} \mathrm{C}$ to $63{ }^{\circ} \mathrm{C}$ and from $25{ }^{\circ} \mathrm{C}$ to $56{ }^{\circ} \mathrm{C}$, respectively. This partly shows that the energy of the acoustic waves generated in the $\mathrm{KO}$ medium was higher and stronger than that in the DO medium, and the higher viscosity of $\mathrm{KO}$ may also contribute to DO's lower increase in temperature. Thus, these results indicate that the choice of appropriate solvent is critical, and this agrees with the conclusions of other studies [5] and [25].

According to the results presented in Fig. 5, it can be observed that the temperature increased along with the increasing ultrasound irradiation time, and at the later time the temperature tends to rise more gradually compared with the previous increase. This slowing rate of change is most probably because of the increasingly high temperature; as the temperature difference between the solvent and the environment increases, more heat is lost to the environment. Then at a certain point the heat loss will be balanced with the heat generated during the ultrasound. At that point, the temperature of the ultrasound solvent will not increase anymore. 


\subsubsection{Variation of pressure drop over ultrasonic time}

Ultrasonic cleaning results are expressed through the parameters of the pressure loss (drop) across the filter. A smaller loss in pressure indicates a cleaner filter, thus reducing operating costs and minimising by-products. Pressure loss value measurements over the time of the ultrasound treatment are shown in Fig. 5, where the results show that increasing the ultrasound irradiation time produces a continuing decrease in the pressure losses through the filter. This means that the effectiveness of the cleaning process is directly proportional to the ultrasonic time. Also, the effectiveness of the ultrasonic cleaning method when KO medium was used on the filters was greater than when a medium of DO was used. This difference can be explained by the fact that the viscosity of $\mathrm{KO}$ is higher than that of DO.

The fouled oil filters were first briefly washed with the manual method, after which the pressure loss of the filter remained at $2940 \pm 3.54 \mathrm{~Pa}$. After $90 \mathrm{~min}$ of ultrasound, pressure loss through the filter had a residual value of $2618 \pm 8.68 \mathrm{~Pa}$ for $\mathrm{KO}$ solvent and $2641 \pm 1.87 \mathrm{~Pa}$ for DO solvent. It can be concluded that optimal ultrasonic irradiation time to achieve the level of satisfaction in the medium of $\mathrm{KO}$ is $50-60 \mathrm{~min}$, whereas in the medium of DO it is above $80 \mathrm{~min}$. For example, after $60 \mathrm{~min}$ of ultrasonic irradiation, the pressure loss through filters in the medium of $\mathrm{KO}$ was reduced to $2628 \pm 8.676 \mathrm{~Pa}$, while for filters treated in the medium of DO the pressure loss only fell to $2682 \pm 1.867 \mathrm{~Pa}$. These different results, observed under the same experimental conditions, indicate that the cleaning efficiency of the ultrasonic treatment system will vary when different solvents are used.

This variation could be attributed to these solvents' different physical characteristics, such as molecular weight, viscosity, surface tension, and vapour pressure [25]. In addition, Pearson correlations were also calculated and results demonstrated a strong correlation between the temperature of the solvent, the pressure loss through the filter and ultrasonic irradiation time, respectively shown in Fig. 5, via a very high correlation coefficient $r$ and significance level $p(p<0.001)$. Furthermore this correlation followed first order exponential decay.

\subsubsection{Corrosion ability of solvent}

To compare the ability of the ultrasound to corrode copper foil in solvents of DO and KO, a preliminary assessment in accordance with method ASTM D130-04 [21] was implemented. This was done by comparing the corrosion of copper foil in the solvents before and after ultrasound was applied. The before and after results for solvent of $\mathrm{KO}$ were $1 \mathrm{~A}$ and $1 \mathrm{~A}$, and for the DO solvent were $1 B$ and $1 B$.

The results showed the corrosion of the copper foil in KO medium at a level of $1 A$, the lowest level of corrosion corresponding to the standard method of ASTM D13[21]. KO showed corrosion levels remained almost constant during the experiment. Meanwhile, for DO medium, copper foil corrosion was at a level of $1 B$, a higher level than $\mathrm{KO}$ medium but this was not significant. Also, DO solvent was not denatured or altered after ultrasound, suggesting that the possible alterations in either solvent have little to no effect on the filter during ultrasound.

Furthermore, the results obtained also indicate that, over the same period of time for ultrasound, filters located in the cleaning solvent of $\mathrm{KO}$ were cleaned far faster than those in the cleaning solvent of DO. It can be stated that the solvent more suitable for ultrasonic cleaning according to this study is KO. According to the evidence outlined above and in other studies, it seems that $\mathrm{KO}$ has good cavitating properties and consequently was chosen as the solvent for the following experiments. 


\subsection{Effects of power ultrasound}

\subsubsection{Variation of temperature in solvent over ultrasound irradiation time}

When step 2 was executed, in the KO medium the solvent temperature in the ultrasonic devices of $300 \mathrm{~W}$ and $600 \mathrm{~W}$ were directly measured with thermometers during the experiment and recorded every $10 \mathrm{~min}$. Measurement results are compared and shown in Fig. 6a.

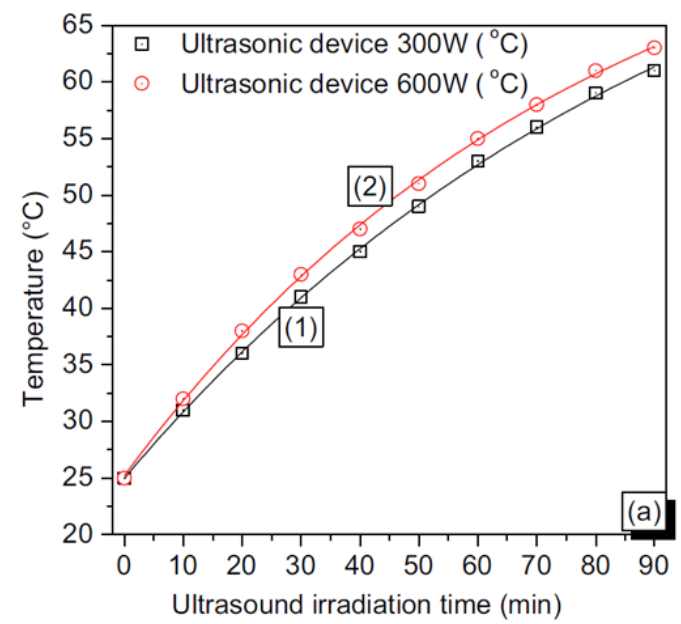

(1): $y=85.58-60.59^{*} \exp (-x / 98.24) ; \operatorname{adj} . R^{2}=0.999$ $(r=0.992, p<0.001)$

(2): $y=80.99-55.77^{*} \exp (-x / 79.05) ;$ adj. $R^{2}=0.999$ $(r=0.988, p<0.001)$

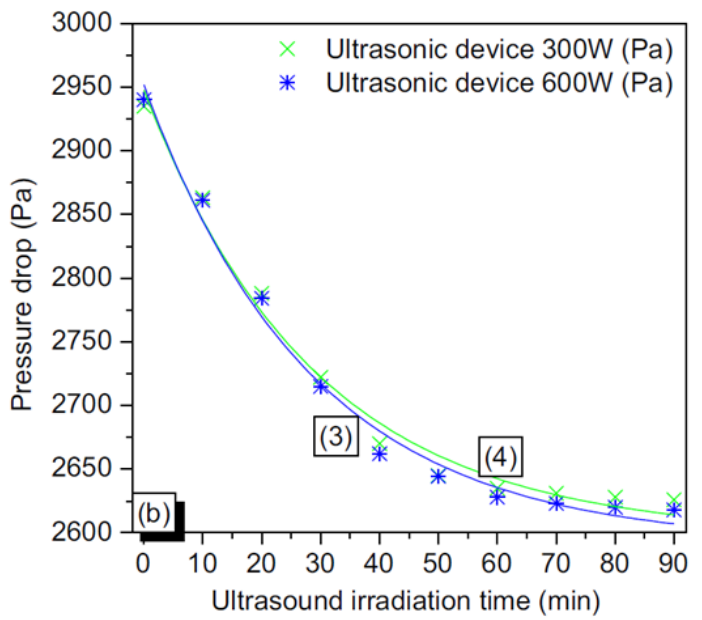

(3): $y=2591.96+359.74^{*} \exp (-x / 28.38) ;$ adj. $R^{2}=0.987$ $(r=-0.906, p<0.001)$

(4): $y=2598.67+349.08^{*} \exp (-x / 28.91) ; \operatorname{adj} . R^{2}=0.984$ $(r=-0.906, p<0.001)$

Fig. 6. Comparing the variation of temperature and pressure loss in a solvent of $\mathrm{KO}$ over ultrasound irradiation time, using ultrasonic devices of $300 \mathrm{~W}$ and $600 \mathrm{~W}$.

Experimental results revealed that the temperature of the solvent in the ultrasonic system is proportional to the duration of the ultrasound treatment and follows first order exponential decay, with adjusted $R^{2}>0.98$ (Fig. 6a). The solvent temperature variations in both experiments slowly increased in similar ways during the ultrasound. However, the degree of increase of the solvent temperature in the ultrasonic device of $300 \mathrm{~W}$ was slower than in the ultrasonic device of $600 \mathrm{~W}$, but not significantly; this difference was only approximately $1.9 \pm 0.33{ }^{\circ} \mathrm{C}$. The temperature increases in the ultrasonic solvents over time were due partly to the minute cavitation bubbles imploding and thus releasing heat energy.

In addition, when analysing the Pearson correlation coefficient, a very strong significant correlation was found between temperature and ultrasound irradiation time. For the ultrasound device of $300 \mathrm{~W}$ the values are $r=0.992, p=2.13 \times 10^{-8}, n=10$, and for the ultrasound device of $600 \mathrm{~W}$ the values are $r=0.987, p=1.05 \times 10^{-7}, n=10$.

\subsubsection{Variation of pressure loss over ultrasound irradiation time}

The results are presented in Fig. $6 \mathrm{~b}$ in order to compare the variation of pressure loss through the filters during the ultrasound, under the same experimental conditions and at optimal frequency for implementing cleaning of $25 \mathrm{kHz}$ [26], with two ultrasound devices of $300 \mathrm{~W}$ and $600 \mathrm{~W}$, respectively. The results illustrated in Fig. $6 \mathrm{~b}$ indicate a strong correlation between pressure loss through the filters and ultrasound irradiation time, almost compatible 
with a first order exponential decay equation. The correlation was expressed through values $R^{2}=0.987, r=-0.906, p=3.043 \times 10^{-4}$ for the ultrasound device of $300 \mathrm{~W}$, and $R^{2}=0.984$ and $r=-0.906$ and $p=3.066 \times 10^{-4}$ for the ultrasound device of $600 \mathrm{~W}$.

Furthermore, through the experimental results observed in Fig. 6b, it seems that the varying pressure losses through the filters when washed in ultrasonic devices of $600 \mathrm{~W}$ and $300 \mathrm{~W}$ were relatively similar. This similarity may be ascribed, although the total power capacity of each device $(600 \mathrm{~W}$ and $300 \mathrm{~W})$ was different, to the devices' parity in terms of specific capacity (both devices had a specific capacity of approximately $100 \mathrm{~W} / \mathrm{gal}$ ). Also, the decrease over time of the pressure losses through the filter may be explained by the collapse of cavitation bubbles, which then generates sufficient force to act on the dirt and dislodge it from the filter.

Consequently, these results suggest that under operational conditions at a constant frequency of $25 \mathrm{kHz}$, and in $\mathrm{KO}$ medium, the ultrasonic irradiation time can be manipulated in order to satisfy filter quality requirements after cleaning.

\subsection{Evaluating the effectiveness of cleaning filters and determining the optimal exposure time}

\subsubsection{Filter cleaning efficiency by using ultrasonic devices}

In order to evaluate the effectiveness of a new method of cleaning oil filters with the assistance of ultrasonic waves, sample filters were treated by ultrasonic devices in a medium of KO. The research group measured the pressure losses through the filters: (1) after a group of filters was washed by hand by a mechanic (Group 1), and (2) after a group of filters had just been taken out of the housing and had received only a preliminary wash to remove any obvious plaque deposits on the filters' surface (Group 2). Both Group 1 and Group 2 were then immersed in $\mathrm{KO}$ and treated with ultrasound waves by a $600 \mathrm{~W}$ ultrasonic generator; afterward the pressure loss remaining in each group was measured to compare with manualonly washing methods.

To minimise margin of error, the test method was carried out on two groups of filters, each having the same characteristics (same type of filters, taken from the same filter housing). The results that were obtained consequently proved to be highly reliable. Then, to evaluate the difference in effectiveness between these modes of washing (hand washing, preliminary washing and ultrasonic washing). This process aimed to confirm the filters' cleanliness after manual washing and ascertain whether filters would benefit from being washed again by ultrasound waves or not. The results obtained (shown in Fig. 7), indicate there is a clear difference between these modes of cleaning. In fact the ultrasonic cleaning method cleans the filters more thoroughly and effectively than traditional manual methods.

As the results show in Fig. 7, the pressure loss through the filter declined from $3538 \pm 8.49$ to $2970 \pm 8.48 \mathrm{~Pa}$ after the filter was subjected to hand washing. Meanwhile, the preliminary washing mode caused the pressure loss to decrease from $3536 \pm 11.55$ to $3154 \pm 9.302 \mathrm{~Pa}$. Next, the same filters were washed by ultrasound for $90 \mathrm{~min}$, with the pressure losses afterwards remaining at $2618 \pm 8.48 \mathrm{~Pa}$ (for the filter that started with manual washing) and $2620 \pm 4.59 \mathrm{~Pa}$ (for the filter that started with preliminary washing). Thus, it can be seen that washing by hand (either preliminary or manual washing) can only detach a very limited amount of the foulant particles deposited on the filter surface and seems unable to thoroughly clean away the residue formed in the slots, corners, or tiny spaces of the filters. Moreover, the evidence above confirms that with continuous ultrasound washing of just 50-60 min at most, the filters reach an acceptable value (selected as the optimal value) of pressure loss. 50 
60 min of ultrasound irradiation was deemed optimal, because the increasingly small progress made after 60-70 min of ultrasound operation was deemed not significant.

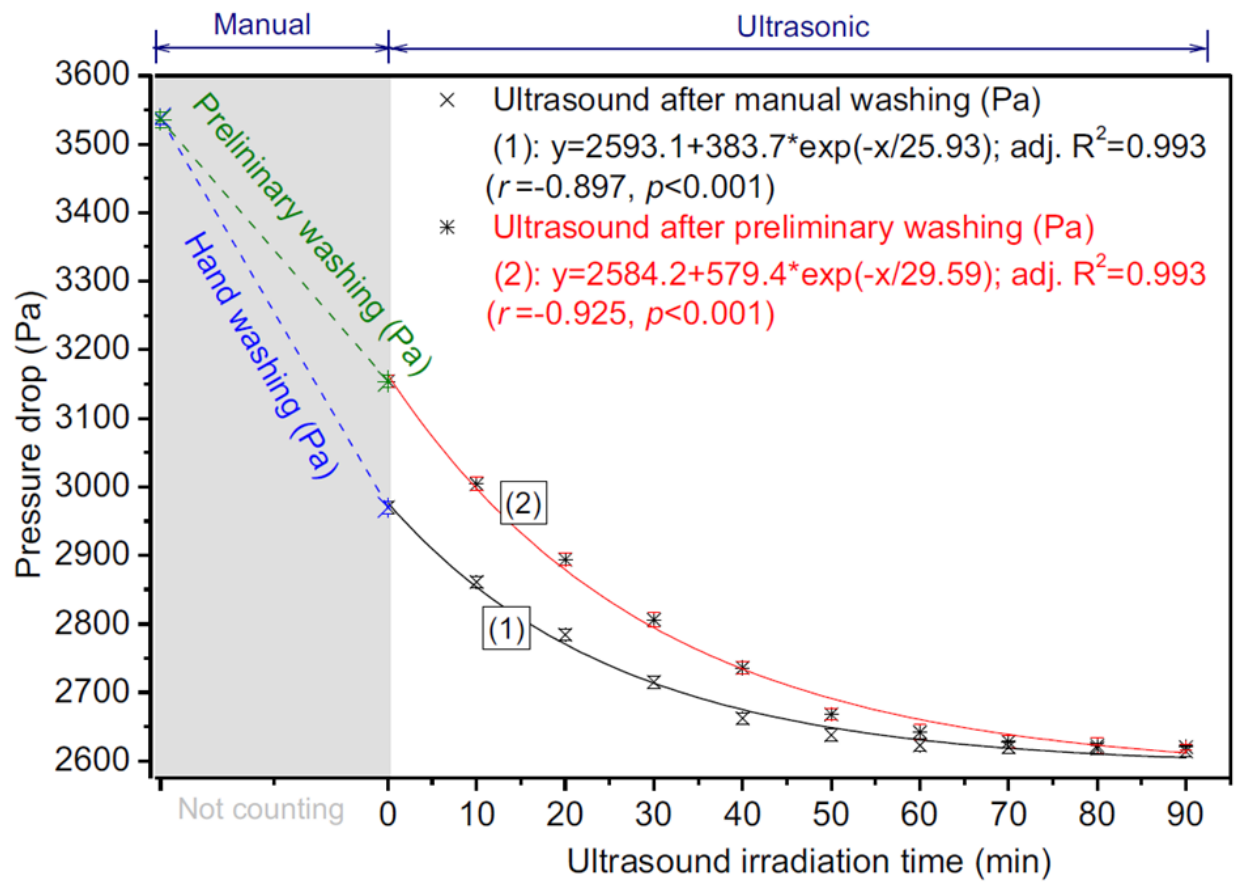

Fig. 7. Comparing the pressure losses over time between these modes of oil filter cleaning.

Test results also showed that ultrasonic washing may be deemed necessary, because it is a more effective, environmentally friendly, and faster method than the conventional manual washing methods still being employed on Vietnam navy ships. This greater effectiveness can also be explained due to the fact that manual washing methods are limited to the use of largescale tools such as mops and brushes, so manual washing only works to clean the adhesive plaque on the surface of the filter and cannot effectively clean joints, slots, niches, corners, or foulants with abnormal adhesive strength on the filter. However, the pulse waves generated by the ultrasonic process can pass through or drive deep inside the filter and provide sufficient force to degrade or dissolve a variety of stains in every part of the filters into the solvent oil, so that the foulants may be flushed out; moreover, the filters can bear even the greatest ultrasonic force applied without damage.

\subsubsection{Optimal duration of ultrasonic cleaning}

It was observed that the efficiency of the filter cleaning process gradually increased with increasing ultrasonic irradiation time in $\mathrm{KO}$ solvent. The optimal effective duration of ultrasonic irradiation with ultrasonic cleaning electrical power of $600 \mathrm{~W}$ for the filters that were manually washed ranged from 50 to $60 \mathrm{~min}$. Conversely the optimal duration for the filters that underwent only preliminary washing ranged from 60 to $70 \mathrm{~min}$ (Fig. 7).

\section{Conclusions}

Considering this study's results for cleaning turbine engines' micro oil filters, it is evident that conventional manual washing methods are not the best choice in terms of efficiency, economy and environmental friendliness. Instead, multi-transducer ultrasonic cleaning could be a reliable technology to radically reduce the problems caused by foulant particles in filters. 
After each cleaning, microscopic observation indicated that no damage had been done to the filter surfaces during the high intensity, low frequency $(\mathrm{kHz})$ ultrasound treatments. In addition, the reduction of pressure loss through the oil filter reached maximum levels with longer ultrasonic irradiation. The multi-transducer ultrasonic device functions by generating unstable cavitation bubbles in the solvent, as the result of the propagation of acoustic waves within the solvent under consecutive compression and expansion cycles (acoustic oscillation). It is the creation and collapse of these miniscule bubbles which dissolves the foulant particles in the solvent and allows them to be removed from the filter. Thus, the use of ultrasonic waves for cleaning turbine engines' oil filters is an attractive option. They demonstrate a more efficient, reliable, and faster washing process than conventional manual washing methods.

Although the oil filters had different initial contaminations, after manual washing the pressure loss through the filter was only reduced by a very limited amount, remaining at about 2940-2976 Pa. After being washed manually, these filters continued to be cleaned by ultrasound, and the pressure loss continued to decrease significantly (by more than $300 \mathrm{~Pa}$ ). The optimal time for which to wash oil filters using this ultrasound method was about $60 \mathrm{~min}$. These results have promising commercialisation possibilities in applying ultrasonic waves to clean oil filters in particular and machine parts in general.

\section{Acknowledgements}

This work was supported in part by Grants from ITE, Viet Nam and Korea Ministry of Environment as "Global Top Project" (Project No: GT-11-B-02-008-3). Dr. M.C. Tran and Dr. N.D. Nguyen and technicians from ITE, Viet Nam are gratefully acknowledged.

\section{References}

[1] J.S. Bartos, R.J.S. Onge, Cleaning and preservation unit for turbine engine, in: Google Patents, 1977.

[2] N. Eliaz, G. Shemesh, R. Latanision, Hot corrosion in gas turbine components, Eng. Fail. Anal. 9 (2002) 31-43.

[3] D. Baumann, N. Prinz, Filter for cleaning lubricating oil, in: Google Patents, 1990.

[4] W. Siegert, Microbial contamination in diesel fuel-are new problems arising from biodiesel blends, in: Proceedings of the 11th International Conference on the Stability and Handling of Liquid Fuels, 2009, pp. 18-22.

[5] T.J. Mason, Ultrasonic cleaning: an historical perspective, Ultrason. Sonochem. 29 (2015) $519-523$.

[6] D. Feng, J.S.J. van Deventer, C. Aldrich, Ultrasonic defouling of reverse osmosis membranes used to treat wastewater effluents, Sep. Purif. Technol. 50 (2006) 318-323.

[7] K.S. Suslick, Ultrasound: Its Chemical, Physical, and Biological Effects, VCH Publishers, 1988.

[8] M.O. Lamminen, H.W. Walker, L.K. Weavers, Cleaning of particle-fouled membranes during cross-flow filtration using an embedded ultrasonic transducer system, J. Membr. Sci. 283 (2006) 225-232.

[9] T.J. Mason, J.P. Lorimer, Applied sonochemistry, Uses Power Ultrasound Chem. Process. (2002) 1-48. 
[10] N.S.M. Yusof, B. Babgi, Y. Alghamdi, M. Aksu, J. Madhavan, M. Ashokkumar, Physical and chemical effects of acoustic cavitation in selected ultrasonic cleaning applications, Ultrason. Sonochem. 29 (2015) 568-576.

[11] T. Leighton, The Acoustic Bubble, Academic press, 2012.

[12] M.O. Lamminen, H.W. Walker, L.K. Weavers, Mechanisms and factors influencing the ultrasonic cleaning of particle-fouled ceramic membranes, J. Membr. Sci. 237 (2004) 213-223.

[13] F. Wang, Y. Wang, M. Ji, Mechanisms and kinetics models for ultrasonic waste activated sludge disintegration, J. Hazard. Mater. 123 (2005) 145-150.

[14] S. Manickam, V.N.D. Arigela, P.R. Gogate, Intensification of synthesis of biodiesel from palm oil using multiple frequency ultrasonic flow cell, Fuel Process. Technol. 128 (2014) 388-393.

[15] J. David, N. Cheeke, Fundamentals and applications of ultrasonic waves, Physics Department Concordia University Montreal, Quebec, Canada, 2002.

[16] M. Sivakumar, S.Y. Tang, K.W. Tan, Cavitation technology - a greener processing technique for the generation of pharmaceutical nanoemulsions, Ultrason. Sonochem. 21 (2014) 2069-2083.

[17] P. Vasiljev, S. Borodinas, R. Bareikis, A. Struckas, Ultrasonic system for solar panel cleaning, Sens. Actuators A 200 (2013) 74-78.

[18] J. Li, R.D. Sanderson, E.P. Jacobs, Ultrasonic cleaning of nylon microfiltration membranes fouled by Kraft paper mill effluent, J. Membr. Sci. 205 (2002) 247-257.

[19] C. Loderer, D. Pawelka, W. Vatier, P. Hasal, W. Fuchs, Dynamic filtration - ultrasonic cleaning in a continuous operated filtration process under submerged conditions, Sep. Purif. Technol. 119 (2013) 72-81.

[20] G. Mazue, R. Viennet, J.Y. Hihn, L. Carpentier, P. Devidal, I. Albaïna, Large-scale ultrasonic cleaning system: Design of a multi-transducer device for boat cleaning (20 kHz), Ultrason. Sonochem. 18 (2011) 895-900.

[21] ASTMD130, Standard Test Method for Corrosiveness to Copper from Petroleum Products by Copper Strip Test, ASTM International, West Conshohocken, 2010. www.astm.org.

[22] W. Colclough, The chemistry of solvents used for ultrasonic cleaning, Ultrasonics 6 (1968) 21-23.

[23] M. Furuta, M. Yamaguchi, T. Tsukamoto, B. Yim, C.E. Stavarache, K. Hasiba, Y. Maeda, Inactivation of Escherichia coli by ultrasonic irradiation, Ultrason. Sonochem. 11 (2004) 57-60.

[24] F.J. Fuchs, Ultrasonic Cleaning, Fundamental Theory and Application, NASA Conference Publication, NASA, 1995. pp. 369-369.

[25] O.A. Antony, Technical aspects of ultrasonic cleaning, Ultrasonics 1 (1963) 194-198.

[26] G. Harvey, A. Gachagan, T. Mutasa, Review of high-power ultrasound industrial applications and measurement methods, IEEE Trans. Ultrason. Ferroelectr. Frequency Control 61 (2014) 481-495.

[27] ASTMD93, Standard Test Methods for Flash Point by Pensky Martens Closed Cup Tester, ASTM International, West Conshohocken, 2010. www.astm.org. 
[28] ASTMD56-05, Standard Test Method for Flash Point by Tag Closed Tester, ASTM International, West Conshohocken, 2010. www.astm.org.

[29] ASTMD86-05, Standard Test Method for Distillation of Petroleum Products at Atmospheric Pressure, ASTM International, West Conshohocken, 2008. www.astm.org.

[30] ASTMD2622-05, Standard Test Method for Sulfur in Petroleum Products by Wavelength Dispersive X-ray Fluorescence Spectrometry, ASTM International, West Conshohocken, 2005. www.astm.org.

[31] ASTMD4294-06, Standard Test Method for Sulfur in Petroleum and Petroleum Products by Energy Dispersive X-ray Fluorescence Spectrometry, ASTM International, West Conshohocken, 2006. www.astm.org.

[32] ASTMD1322-02, Standard Test Method for Smoke Point of Kerosine and Aviation Turbine Fuel, ASTM International, West Conshohocken, 2002. www.astm.org.

[33] ASTMD130-04e1, Standard Test Method for Corrosiveness to Copper from Petroleum Products by Copper Strip Test, ASTM International, West Conshohocken, 2004. www.astm.org. 